\title{
A 10-Gbit/s all-optical code generation and recognition system based on a hybrid approach of optical fiber delay line and superstructure fiber Bragg grating technologies
}

\author{
P. C. Teh, J. H. Lee, M. Ibsen, P. Petropoulos, and D. J. Richardson \\ Optoelectronics Research Centre, University of Southampton, Southampton SO17 1BJ, UK
}

We demonstrate a hybrid all-optical $160 \mathrm{Gchip} / \mathrm{s}$ encoder-decoder system based on fiber delay lines and a SSFBG. Our experiments confirm the possibility of using this approach in reconfigurable pointto-point optical correlation systems.

\section{Introduction}

All optical generation and recognition of packet address is a key requirement for future high capacity optical networks. Recently optical code division multiple access (OCDMA) technology was adopted for header recognition in optically routing data packets, and was proved to be a reliable approach [1]. The main issue related to the OCDM approach though is how reliably the optical signals of the code sequences can be generated and recognized at the transceiver ends.

A number of approaches for generating optical code signals have been reported to date, these include; arrays of optical fiber delay line [2], planar lightwave circuits (PLCs) [3], arrayed waveguide gratings (AWGs) [4], and fiber grating based devices (FBGs) [5,6]. We have previously demonstrated the use of superstructure fiber Bragg gratings (SSFBGs) as an alternative approach for encoding/decoding [6]. All experiments reported so far have used identical technologies for implementing both the encoder and decoder. However, inter-applicability between different approaches, which means dissimilar implementation methods used at the encoder/decoder ends has not yet been demonstrated and analyzed. In this paper, we address this compatibility issue between code generating and recognizing schemes, and demonstrate for the first time that SSFBGs can be used to recognize code sequences generated from an optical fiber delay line based encoder.

\section{Experimental setup and results}

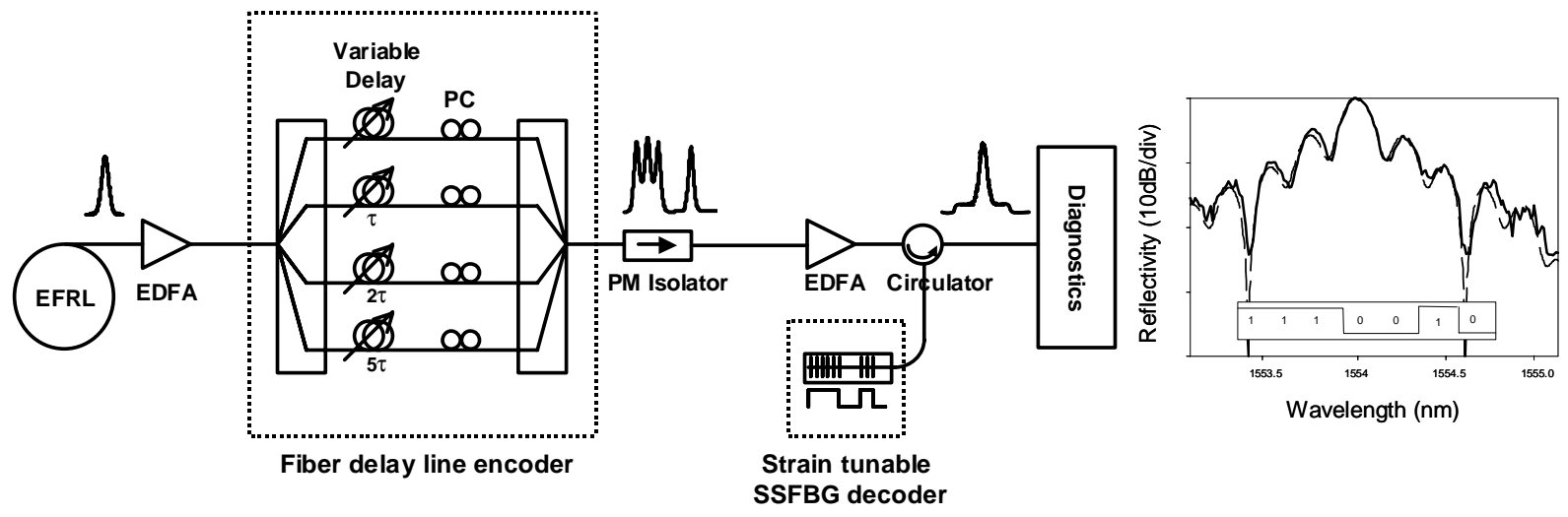

Fig. 1: Experimental setup and SSFBG reflectivity profile; where EFRL: erbium doped fiber ring laser, PC: polarization controller, PM isolator: polarization maintaining isolator, SSFBG: superstructure fiber Bragg grating

Fig. 1 shows the experimental set up used to test this hybrid approach. A $10 \mathrm{GHz}$ regeneratively mode locked erbium fiber ring laser (EFRL) producing 2ps pulses is used as the source. The operating wavelength of the laser is $1554 \mathrm{~nm}$ corresponding to the center wavelength of the SSFBG. The pulses are launched into the optical fiber delay line encoder through a splitter to generate the desired code. As the decoder, a SSFBG also operating as a matched filter [7], is used to recognize the encoded sequence. The resulting pulse form after reflection from the decoder 
grating is measured and analysed using both a fast photodiode/scope ( $25 \mathrm{GHz}$ bandwidth) and an autocorrelator (<100fs resolution).

The optical fiber delay line encoder consists of four parallel fiber delay arms, where each delay is set according to a 7-chip M-sequence amplitude code (1110010). Each delay $\tau$ corresponds to $6.4 \mathrm{ps}$, hence a chip rate of 160 Gchip/s, and the encoded sequence has a total duration of $44.8 \mathrm{ps}$. The M-sequence code is chosen so that the autocorrelation features upon decoding will have a single dominant, well-defined peak with low level cross correlation features. A polarization controller (PC) in each delay line is used to align the individual pulses to a single polarisation axis. The delayed pulses are then recombined and launched into a polarisation maintaining (PM) isolator to confirm the same polarization state of the composite delayed pulses.

The 7-chip amplitude modulated SSFBG decoder was written using our continuous grating writing technique as reported in [8]. It has a total length of $4.62 \mathrm{~mm}$ with an absolute reflectivity of $\sim 4 \%$. The individual chip duration is $6.4 \mathrm{ps}$ corresponding to a chip rate of $160 \mathrm{Gchip} / \mathrm{s}$. The measured reflectivity profile of the SSFBG decoder is shown as in inset to Fig. 1 (solid line) and shows good agreement with the theoretical calculation (dashed line).

In order to quantify the quality of the fiber delay line encoder, we performed a series of code generation experiments to examine the temporal characteristics of the pulse forms generated from the fiber delay line. These results then were compared to the pulse form generated by using a SSFBG as the decoding element. Fig. 2a shows the temporal response of the fiber delay line encoder as measured using the SHG autocorrelator as well as the direct electronic measurements on the oscilloscope with $\sim 25 \mathrm{GHz}$ spectral bandwidth. The measured autocorrelation and oscilloscope traces of encoded signals are found to be in good agreement with the theoretical predictions confirming not only the formation of the correct code patterns but also the desired individual chip separation of 6.4ps. Fig. $2 \mathrm{~b}$ shows the equivalent results of the pulse forms on reflection from the SSFBG decoder (in this case used as the encoder) with evidence of good qualitative coincidence between experiment and theory.

(a) Delay line Encoder
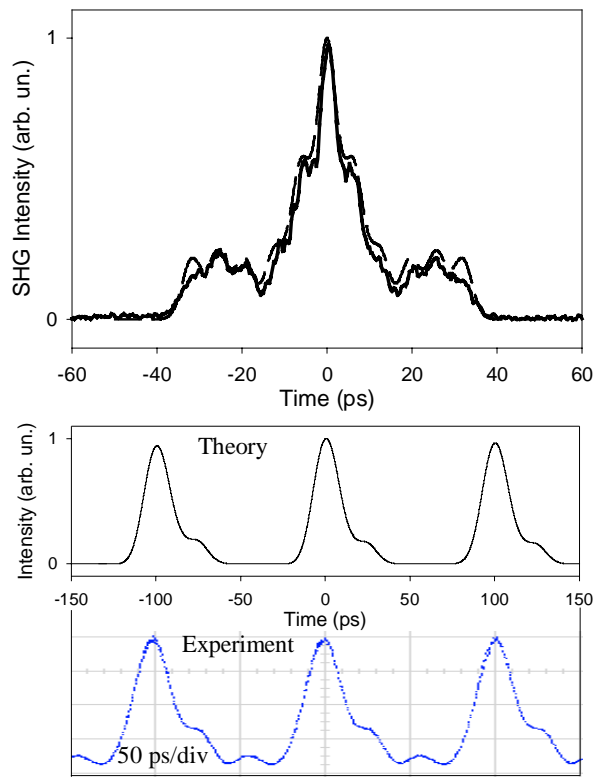

(b) SSFBG Encoder
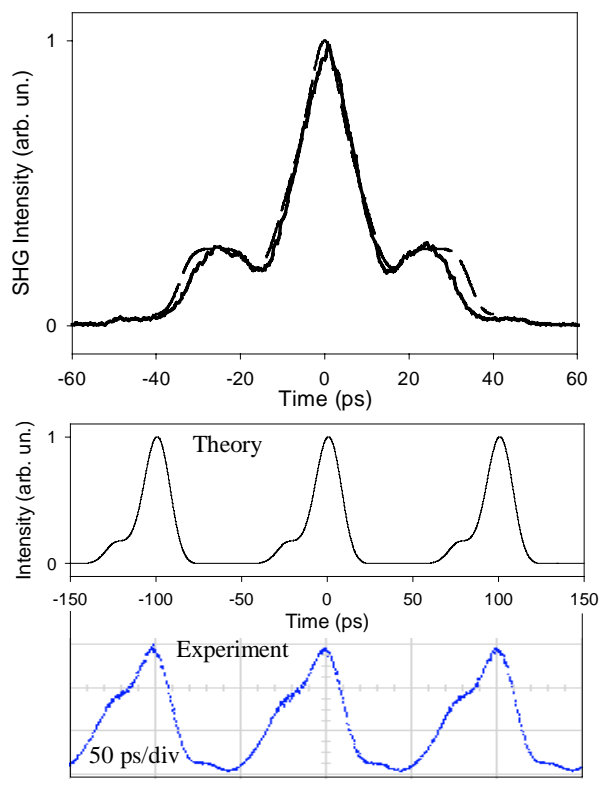

Fig. 2: (a) Delay line encoder (b) SSFBG encoder

Above: Intensity SHG autocorrelation traces of the encoded sequence (solid line: experiment, dashed line: theory) at the output of the particular encoder.

Below: Theoretical and experimental traces of the encoded waveform at $10 \mathrm{Gbit} / \mathrm{s}$ with $25 \mathrm{GHz}$ bandwidth limitation included.

(Note: the SSFBG encoder used in (b) is being reflected from the opposite side and hence the time inversed nature of the encoded waveform.)

To characterize the full hybrid system, the two different schemes of encoding/decoding (Delay line encoder:SSFBG decoder, SSFBG encoder:SSFBG decoder) then were put together and the results of the code recognition analysed and compared. In the case of the fiber delay line encoder, fine control of both the phase and polarization is required to obtain the optimum results. This is because each delayed pulse experiences a random phase change, which can lead to changes in the form of the output recognition pattern [2]. We directly compared the SHG autocorrelations of the code recognition signature of the 7-chip unipolar code against the theoretical predictions and found good agreement between them as shown in Fig. 3a. The well-defined code recognition peak 
was found to have a pulse width of $\sim 12.4$ ps. Despite the limitation of oscilloscope bandwidth, we were able to obtain a single peak deconvolved decoded pulse form with good agreement to the theoretical calculations. These results can be compared to those obtained by SSFBG based encoder and decoder with the equivalent SHG autocorrelation traces shown in Fig. 3b.

(a) Delay line Encoder - SSFBG Decoder
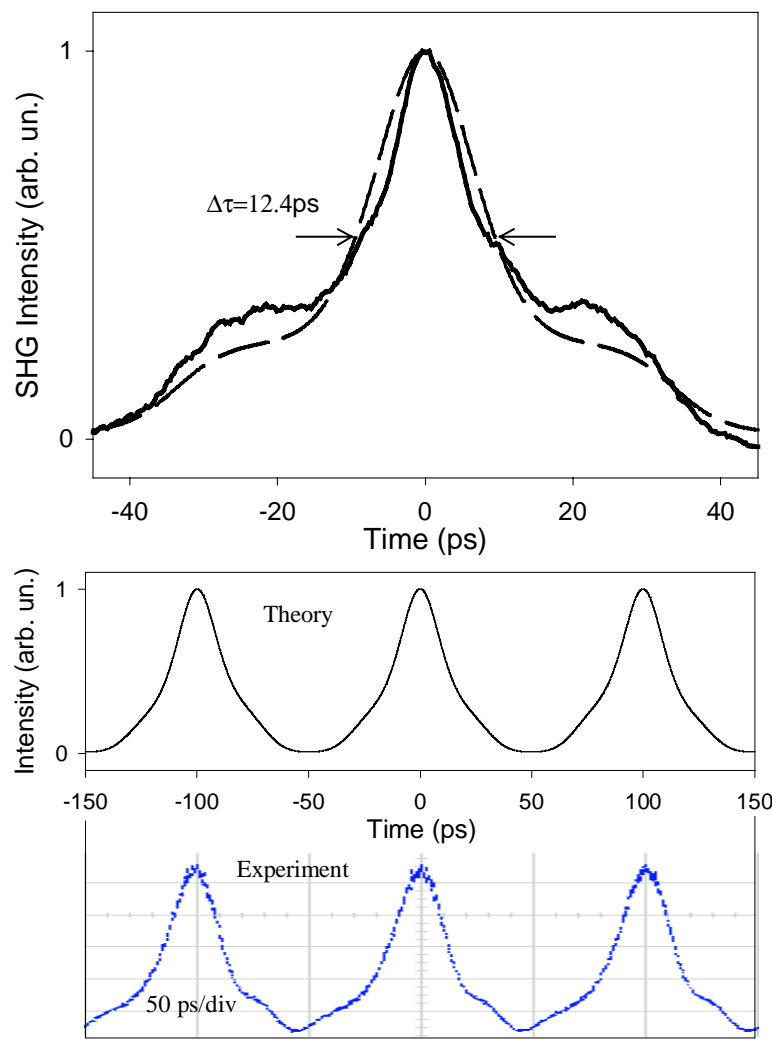

(b) SSFBG Encoder - SSFBG Decoder

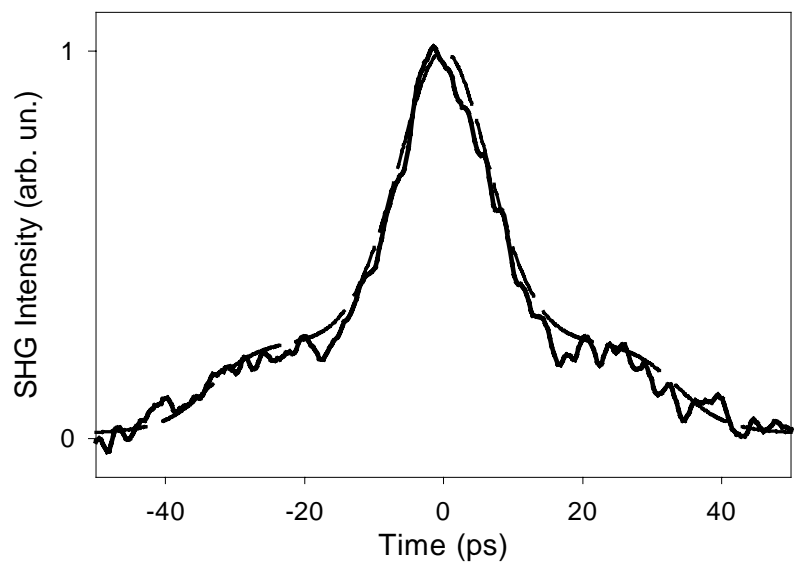

Fig. 3. (a) A fiber delay line encoder - SSFBG decoder system. Above: Intensity SHG autocorrelation traces of the decoded signal (solid line: experiment, dashed line: theory)

Below: Numerically calculated and measured experimental results of the oscilloscope traces of the decoded signal taking into account the $25 \mathrm{GHz}$ bandwidth limitation of the detector at $10 \mathrm{Gbit} / \mathrm{s}$.

(b) A SSFBG based encoder - SSFBG decoder system. Intensity SHG autocorrelation traces of the decoded signal (solid line: experiment, dashed line: theory)

\section{Conclusion}

We have demonstrated both experimentally and theoretically that SSFBGs can be used as an alternating technology to recognize codes generated from another pattern generating scheme, in this case fiber delay lines. These results illustrate that the SSFBG approach is compatible with other technologies for code generation. Our experiment constitutes the first demonstration of a hybrid all-optical encoder-decoder system, an approach that could prove a necessary solution for reconfigurable point-to-point optical correlation systems.

\section{References}

[1] K. Kitayama and N. Wada, "Photonic IP routing," IEEE Photon. Technol. Lett., 11, 1689-1691 (1999).

[2] K. P. Jackson, S. A. Newton, B. Moslehi, M. Tur, C. C. Cutler, J. W. Goodman, and H. J. Shaw, "Optical fiber delay-line signal processing," IEEE Trans. Microwave Theory Tech., MTT-33, 193-209 (1985).

[3] N. Wada, and K. Kitayama,"A 10Gb/s optical code division multiplexing using 8-chip optical bipolar code and coherent detection,"J. Lightwave Technol., 17, 1758-1765, (1999).

[4] H. Tsuda, H. Takenouchi, T. Ishii, K. Okamoto, T. Goh, K. Sato, A. Hirano, T. Kurokawa, C. Amano, "Spectral encoding and decoding of $10 \mathrm{~Gb} / \mathrm{s}$ femtosecond pulses using high resolution arrayed-waveguide grating," Electronics Lett., 35, 1186-1187, (1999).

[5] A. G-. Jepsen, A. Johnson, E. Maniloff, T. Mossberg, M. Munroe, and J. Sweetser,"Spectral phase encoding and decoding using fiber Bragg grating," in Proc. Optical Fiber Communication Conference (OFC'99), PD33-1-3, (1999).

[6] P. C. Teh, P. Petropoulos, M. Ibsen and D. J. Richardson, "The generation, recognition and re-coding of 64-bit, 160 Gbit/s optical code sequences using superstructured fiber Bragg gratings," in Proc. Optoelectronics \& Communication Conference (OECC'2000), PD1-3 (2000).

[7] H. Geiger, M. Ibsen, R. I. Laming, “Optimum receivers with fiber gratings", in Proc. Optical Fiber Communication Conference (OFC'98), 152-154, (1998).

[8] M. Ibsen, M. K. Durkin, M. J. Cole, and R. I. Laming, "Sinc-sampled fiber Bragg gratings for identical multiple wavelength operation,” IEEE Photon. Technol. Lett., 10, 842-844 (1998). 